\title{
Development of Ultra-Broadband Base Station Antenna for All Mainstream LTE 700/800/900 MHz Frequency Bands
}

\author{
Doudou Samb, ${ }^{1}$ Zhonglin Wu, ${ }^{1}$ Mulin Liu, ${ }^{1}$ and Hu Bin Jie ${ }^{2}$ \\ ${ }^{1}$ Department of BSA, Tongyu Communication Inc., Zhongshan 528437, China \\ ${ }^{2}$ Short Distance Detection \& Communication Key Lab of Guangdong Province, South China University of Technology, \\ Guangzhou 510640, China \\ Correspondence should be addressed to Doudou Samb; doudousamb@tycc.cn
}

Received 9 July 2014; Revised 21 October 2014; Accepted 21 October 2014; Published 20 November 2014

Academic Editor: Giacomo Oliveri

Copyright (C) 2014 Doudou Samb et al. This is an open access article distributed under the Creative Commons Attribution License, which permits unrestricted use, distribution, and reproduction in any medium, provided the original work is properly cited.

\begin{abstract}
LTE deployment is being accelerated due to its improved radio access structure meeting the requirements of current and next generation of wireless networks. Its low band application presents useful aspects such as low density of base station while providing good in-building penetration. In this work, we design and develop a dual-polarized base station antenna supporting $698 \mathrm{MHz}$ to $960 \mathrm{MHz}$ with an azimuth-plan half-power beam width of $90^{\circ}$ covering all mainstream LTE 700/800/900 MHz frequency bands representing the widest low frequency range being actively used in the current mobile communication industry. In the design process, rigorous algorithm based on swarm method is developed to tune the electrical performances under strict base station antenna requirements. Experimental results from pattern tests demonstrate the design analysis and the significant advantages of using swarm method in the antenna development process.
\end{abstract}

\section{Introduction}

Wireless communication [1-6] access will continue to increase where operators aim to exploit different network applications in which interference cancellation/reduction [2, 5] presents a vital issue for fully spectrum usage in presence of a fading change over time environment. Besides, mobile users are demanding more application services that need high capacity offered through wireless communication systems. However, tracing back the Shannon theory stated the relation between capacity and bandwidth [7]; one can effectively provide high data rate applications through wideband frequency system. As an important fact, operators struggle from extending their existing operating bands while requesting new ones for advanced network applications. And remarkable progress in wireless communications aims not only to satisfy the requirements for current wireless systems but also to advance the state-of-the-art of the communication field putting it in the context of long term evolution (LTE) networks and beyond $[6,8]$. LTE aims to improve the radio access structure to meet the requirements of current and future wireless networks introducing scalable bandwidths varying from $1.4 \mathrm{MHz}$ up to $20 \mathrm{MHz}$ where users can experience high data rate applications up to $100 \mathrm{Mbps}$ in downlink and $50 \mathrm{Mbps}$ in uplink with latency less than $5 \mathrm{~ms}$ useful for real time applications. Interestingly, its integration system is flexible which means that deployment can be carried out progressively alongside regular cellular networks. Accordingly, these fastest emerging wireless systems generate numerous challenges on the antenna system design as new frequency bands should be fully covered to meet the demands of various application levels impacting on the radio network design involving, for example, the propagation and cell planning mechanisms. Consequently, there is extensive investigation about developing ultra-wideband antennas $[9,10]$. Base station antennas (BSAs) are capable of converting energy between electricity and electromagnetic waves and are mainly composed of several radiating elements (dipoles) useful for transmitting/receiving information throughout a communication system. The new $700 \mathrm{MHz}$ band opens up great challenges when one aims to cover the whole frequency range for the entire required LTE low band applications with global relevance. However, LTE's low band features useful aspects such as low density of base station which reduces operator's 
CapEX and OpEX while providing good in-building penetration with good overall coverage performances across the sector. In addition, when designed with a wide beam width in the azimuth-plan (azimuth half-power beam width of $90^{\circ}$ ), one can offer ultra-broadband antenna supporting the widest low frequency range being actively used in the mobile communication industry useful for coverage-oriented networks. A mostly suitable application is low mobility area such as predominantly rural regions. And to date, there is no known ultra-broadband dual-polarized variable down tilt BSA covering 698-960 MHz with 90 deg azimuth beam width. Besides, LTE deployment is being accelerated worldwide.

The present study is the design and development of such antenna covering all mainstream LTE $700 / 800 / 900 \mathrm{MHz}$ frequency bands. The physical structure is set up based on antenna theory analysis. In addition, we develop an optimization algorithm based on particle swarm (PSO) $[11,12]$ method to control the overall pattern performances over the entire operating frequencies depending on requested specifications. Furthermore, the antenna is fabricated and tested within an anechoic chamber and experimental results demonstrate the design analysis and the significant advantages of using swarm method in the antenna development process.

The rest of the paper is organized as follows. Section 2 describes the antenna design specifications. Section 3 presents the structure design and analyzes the horizontal pattern. In Section 4, the vertical pattern is analyzed with the development of a PSO algorithm to find the optimum amplitude and phase of the voltage excitations of the different elements outputting the specified side lobe levels. Section 5 presents the experimental results while Section 6 concludes this work.

\section{Antenna Design Specifications}

The antenna should be dual-polarized $\left( \pm 45^{\circ}\right)$ covering a wide range of $698-960 \mathrm{MHz}$, with a VSWR not greater than 1.5 and a port-to-port isolation not less than $25 \mathrm{~dB}$ with a wider horizontal beam width stable across the whole operating band $\left(90^{\circ} \pm 5^{\circ}\right)$ and a vertical pattern showing a first side lobe level (SLL) greater than a given target. In addition, a medium or higher gain is requested as well within a down tilt range of $0^{\circ}-10^{\circ}$ to control the pattern useful for optimization at the operators' side for example. Consequently, we can affirm that an antenna array should be built. And such array is composed of several radiating elements.

\section{Structure and Horizontal Pattern Analysis}

The radiating element is a crossed-dipole design forming a dual-polarized half-wave dipole useful for base station applications. The radio performances are optimized using High Frequency Structure Simulator (HFSS) software. And since the design of the radiating element is not object of this paper, its structured process is not included (designed separately and is under patented process). Meanwhile, as it is depicted in Figure 1, it is composed of two half-wave length dipoles. It is optimized to output good impedance match with a port-to-port isolation of about $22 \mathrm{~dB}$ and a stable radiation

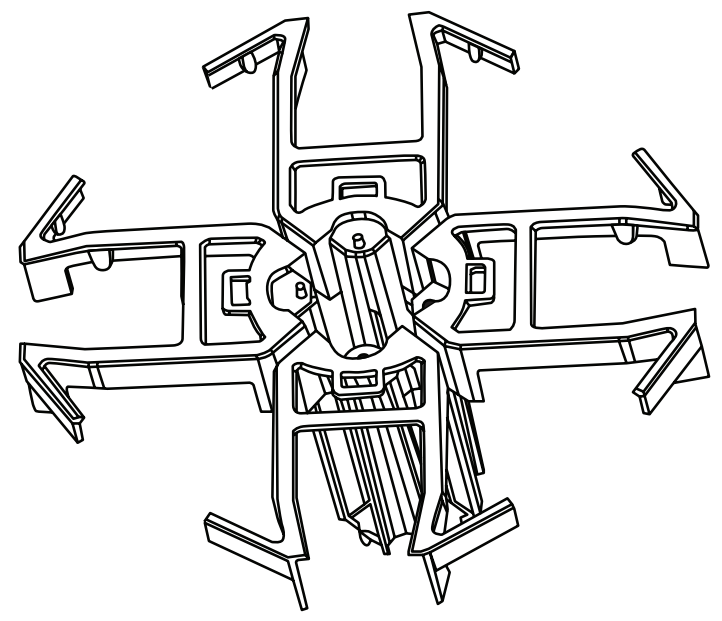

FIgURE 1: Dual-polarized radiating element.

pattern over the entire operating band of frequency as will be shown during the next steps.

However, the wideband characteristics of a base station antenna are related not only to a single element performance, but also to the set of elements forming an array. Therefore, particular considerations should be given to the structure of such array as analyzed in the next parts.

To set up the array, the number of and the distance between two elements are very important and need to be investigated carefully. Interestingly, the minimum number of elements needed can be approximated based on the requested gain. And this can be obtained by knowing the gain of a single element (denoted as $G_{S}$ ) and using the fact that by adding a new element the gain increases by $3 \mathrm{dBi}$ theoretically. It follows that the ideal gain $G_{T}$ and the number of elements $N$ satisfy

$$
G_{T}=G_{S}+10 \log (N) .
$$

The single element used as presented above is characterized by a half-wavelength dipole outputting a gain of about $8 \mathrm{dBi}$ (typically). However, the requested gain is affected by the losses from the different components used to feed the antenna. So, extra margin should be considered to compensate such negative effect. For example, using a margin of about $\pm \varepsilon_{m}$ leads to a theoretical maximum gain based on which an approximate number of elements can be obtained from (1). In addition, the closeness of the radiating elements may affect the cross coupling which may lower the insulation between the $+45^{\circ}$ and $-45^{\circ}$ polarizations. And when the distance between two elements (known as spacing factor) becomes large, extra lobes may appear along with the main beam. Such additional lobes well known in the literature as grating lobes [13] should be considered since they only contribute in terms of wasted power which affect the efficiency of the antenna. The recommended limits propose a value (distance between antenna elements) not lower than half and not greater than full wavelength at the operating frequency (generally chosen to be the center frequency). Based on analysis as will be presented below, we obtain an appropriate value around 


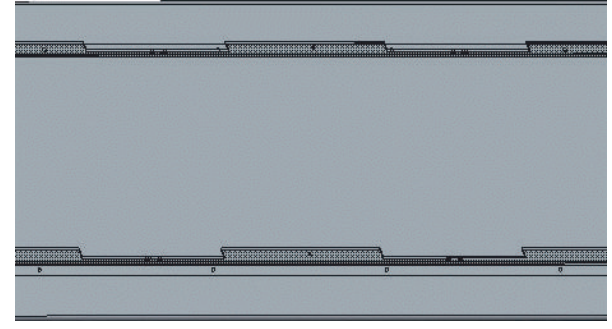

FiguRE 2: Simplified reflector structure for two elements.

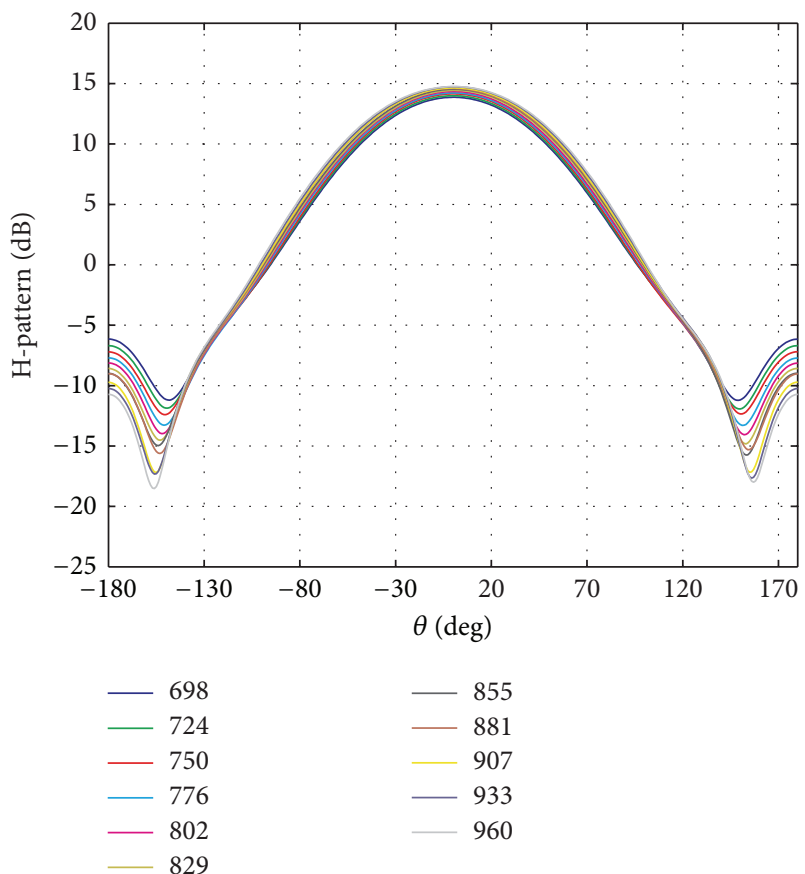

FIGURE 3: Radiation pattern showing the azimuth-plan half-power beam width.

$0.5 \sim 0.8$ times the operating frequency wavelength according to the model studied.

As cellular antenna applications, the elements are positioned on a reflector to get a better control of a broadside radiation pattern. The physical structure of the reflector influences the sectorial characteristics especially in the azimuthplane. For example, to output low back radiation, secondary walls can be added running perpendicular to the antenna array axis. And since the length is related to the interelement spacing and the number of elements, we can optimize its physical structure by determining the appropriate width and height providing the targeted azimuth-plane half-power beam width of $90^{\circ} \pm 5^{\circ}$. To do this, a two-element model is built using HFSS. And a MATLAB application is used as in [14] to find the optimal width of the walls being about $180 \mathrm{~mm}$ with a height of about $54 \mathrm{~mm}$ (simplified reflector is shown in Figure 2). Extra cavities are added around the walls for further tuning and are good for isolation improvements as observed during the simulation process.

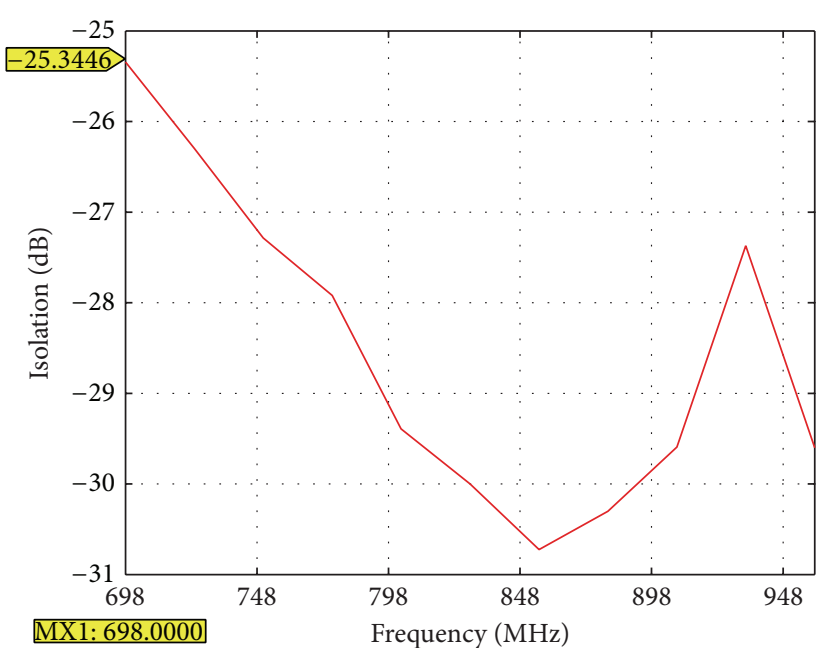

FIGURE 4: Isolation according to the frequency.

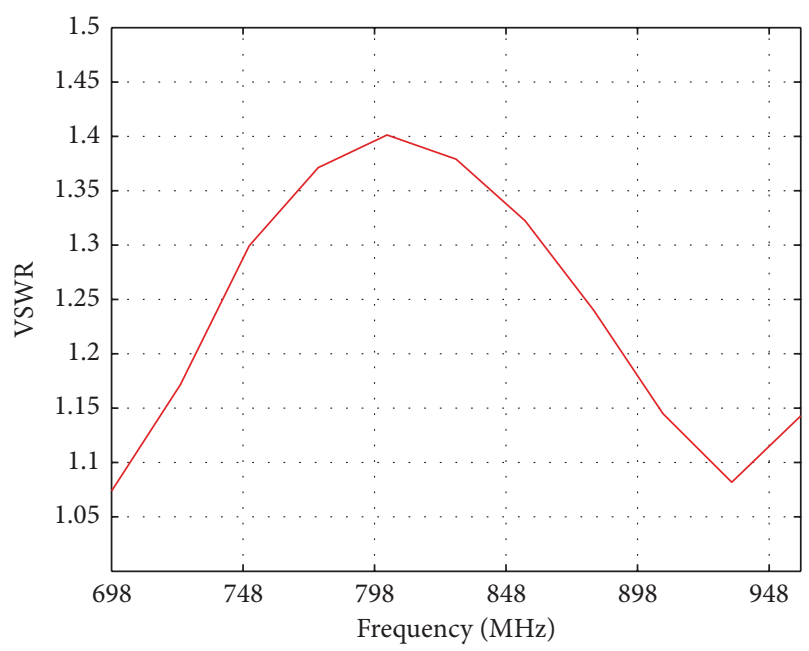

FIGURE 5: VSWR according to the frequency.

By making use of the above remarks and points, we then extend the length of the reflector for five-element model outputting so the simulation results presented in Figures 3, 4 , and 5. From the figures, we can realize a stable radiation pattern, a good port-to-port isolation of about $25 \mathrm{~dB}$, and a VSWR less than 1.5 across the whole frequency range.

\section{Vertical Pattern Analysis}

To address the vertical pattern performances regarding the SLL constraint across the down tilt range of $0^{\circ}-10^{\circ}$, rigorous analysis is conducted as discussed below.

From the array theory, the far-field pattern of the $N$ elements antenna can be obtained by making use of the pattern multiplication principle relating the far-field of a single element and a term named as array factor $[13,15]$. Let $A_{i}$ and $B_{i}$ denote the amplitude and phase of the voltage excitation of the $i$ th element (element being numerated from 1 to $N$ with the first element located at the edge of the array), 
respectively, based on which array factor can be expressed simply as element-by-element sum as

$$
\operatorname{AF}(\theta)=\sum_{i=1}^{N} A_{i} e^{j\left[(i-1) k d \sin (\theta)+B_{i}\right]},
$$

where $d$ is the spacing factor, $k=2 \pi \lambda^{-1}$ represents the wave number, and $\theta$ is the elevation angle generally chosen between $-\pi$ and $\pi$.

Note that the far-field expression in (2) represents a pattern steered at $0^{\circ}$. However, to steer the pattern at a specified angle $\theta_{0}$, the phase shift of each element should be considered as well. The phase shift of the $i$ th element depends on the free space phase shift

$$
\phi_{0}=k d \sin \left(\theta_{0}\right)
$$

and its shifted amount is denoted as $f_{N}(i)$ (chosen from a carefully phase reference position $i_{r}$ with $f_{N}\left(i_{r}\right)=0$ ). Roughly speaking, consider, for example, $N$ elements numerated from 1 to $N$, with a given referenced element $i_{r}$, the phase shift of the $i$ th element is given as

$$
p_{i}=f_{N}(i) \phi_{0} .
$$

For example, let us consider 5 elements numerated as 1, 2, 3, 4 , and 5. By choosing the center-element as phase reference $\left(i_{r}=3\right)$, then the shifted amount of the different elements is given by $f_{5}(1)=-2, f_{5}(2)=-1, f_{5}(3)=0, f_{5}(4)=1$, and $f_{5}(5)=2$, respectively.

Thus, combining (2) and (4) we can express the far-field array factor for a given steered angle $\theta_{0}$ as

$$
\operatorname{AF}_{\theta_{0}}(\theta)=\sum_{i=1}^{N} A_{i} e^{j\left[(i-1) k d \sin (\theta)+B_{i}+f_{N}(i) k d \sin \left(\theta_{0}\right)\right]}
$$

Therefore, the side lobes defined for values of $\theta$ out of the main beam range can be controlled by carefully analyzing expression (5). In fact, we can realize that the problem becomes now to find the amplitude and phase of the voltage excitations of the different elements subject to a required maximum SLL across a steered range. Consequently, by formulating an optimization algorithm, the problem can be analyzed and solved through numerical methods [16-20] among which we chose the particle swarm one as briefly introduced below.

4.1. Concept of PSO Algorithm. Computational technique is introduced in 1995 by Kennedy and Eberhart [18]. It is not only simple compared to genetic algorithm (GA) [20], simulated annealing (SA) [16], and evolutionary algorithms $[17,19]$ but also efficient [12]. PSO is generally based on the coordination of particles (swarms) moving towards a target within a solution space. Each particle can be represented by its position and velocity (initialized randomly) in the solution space. And their movement can be coordinated easily if each of them knows always its best position (named personal best position) and the best position among all the particles (generally called global best position). The position and velocity of the $i$ th particle can be denoted, respectively, as $X_{i}=\left(x_{1}, x_{2}, x_{3}, \ldots\right)$ and $V_{i}=\left(v_{1}, v_{2}, v_{3}, \ldots\right)$. Through a test function (called also fitness function) [3], each particle's position and velocity can be regularly updated using the following equations:

$$
\begin{gathered}
v_{i}=w v_{i}+c_{1} \operatorname{rand}()\left(p_{i}^{\text {best }}-x_{i}\right)+c_{2} \text { rand }()\left(g^{\text {best }}-x_{i}\right), \\
x_{i}=x_{i}+v_{i},
\end{gathered}
$$

where $p_{i}^{\text {best }}$ and $g^{\text {best }}$ represent the $i$ th particle's personal best position and the global best position among all the particles, respectively. $w$ is the inertia weight, $c_{1}$ and $c_{2}$ represent the acceleration constants, and rand() denotes a predefined function that generates a number in $[0,1]$ in a random fashion.

4.2. Pattern Analysis Using PSO. Based on the above points, we can define the different parameters of the different elements based on which PSO algorithm is developed with 10 elements forming a particle (we apply it for a 5-element array but the approach is suitable to analyze a high-order element as well). It follows that the $i$ th particle within the solution space can be defined as

$$
X_{i}=\left(A_{1}, A_{2}, A_{3}, A_{4}, A_{5}, B_{1}, B_{2}, B_{3}, B_{4}, B_{5}\right) .
$$

The validity of a particle being optimal can be guaranteed by carefully defining a test function (fitness function as already mentioned above). As we can notice, the main parameters to be optimized for a given maximum SLL across a steered range are the amplitude and phase of the voltage excitations of the different elements within the operating frequency band. So, for a given number of frequency points, defining a function taking into account the maximum side lobe value within the tilt range $\left(\theta_{0} \in[0,10]\right)$ seems to be a suitable candidate. Without loss of generality, we consider the minimum, medium, and maximum tilt values to be optimized simultaneously, that is, the far-field pattern for $0^{\circ}, 5^{\circ}$, and $10^{\circ}$ tilt. Consequently, we can define a fitness function as

$$
\begin{aligned}
& f(\theta)=\max \left\{\max \left\{\operatorname{SLL}\left(\theta_{\mathrm{SL} 0}\right)\right\}, \max \left\{\operatorname{SLL}\left(\theta_{\mathrm{SL} 5}\right)\right\},\right. \\
& \max \left\{\operatorname{SLL}\left(\theta_{\mathrm{SL} 10}\right)\right\},,
\end{aligned}
$$

where $\theta_{\mathrm{SL} 0}, \theta_{\mathrm{SL} 5}$, and $\theta_{\mathrm{SL} 10}$ represent the region of the side lobes (region out of the main beam) for $0^{\circ}, 5^{\circ}$, and $10^{\circ}$ tilt, respectively.

In addition, the objective function to be optimized can be defined as the sum of the function defined in (5) formed by the three corresponding tilts as

$$
\begin{aligned}
F(\theta)=\sum_{i=1}^{N} A_{i} e^{j\left[(i-1) k d \sin (\theta)+B_{i}\right]} & \\
& \times\left(1+e^{j f_{N}(i) k d \sin \left(5^{\circ}\right)}+e^{j f_{N}(i) k d \sin \left(10^{\circ}\right)}\right) .
\end{aligned}
$$




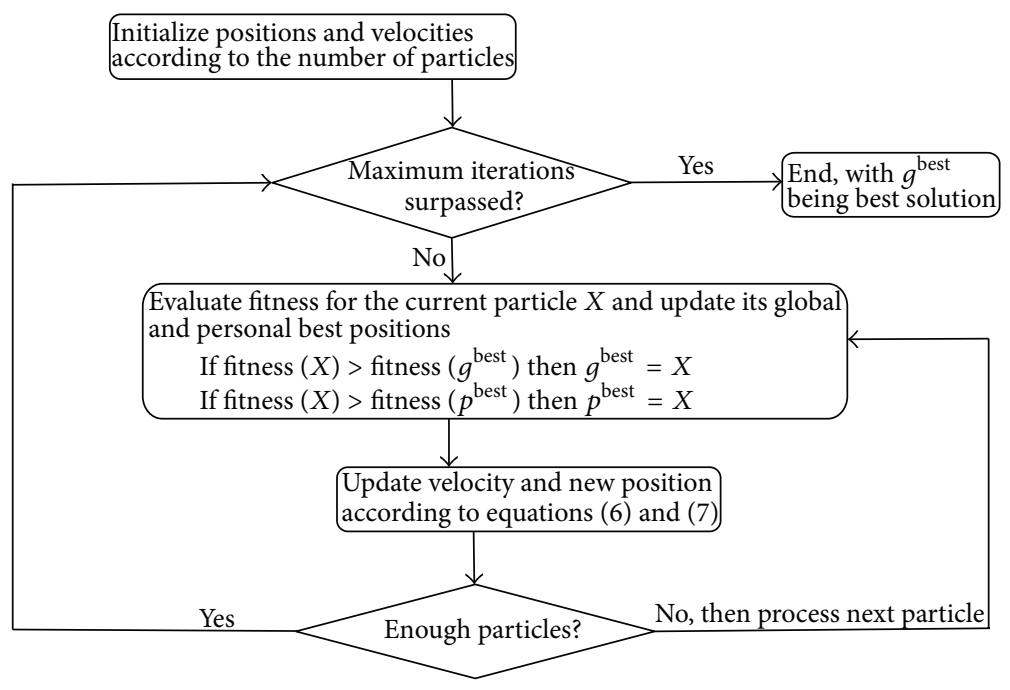

FIGURE 6: Diagram summarizing the PSO process over the iterations.

TABle 1: Amplitude and phase of the voltage excitations of the different elements.

\begin{tabular}{cccccc}
\hline$A_{i}$ & 0.533 & 0.8171 & 1 & 0.8349 & 0.6926 \\
\hline$B_{i}$ & 14.9867 & 4.2445 & 4.4788 & 6.6242 & 3.6329 \\
\hline
\end{tabular}

Therefore the optimization problem can be formulated as

$$
\begin{gathered}
\operatorname{Min} F(\theta), \\
\max \left\{\max \left\{\operatorname{SLL}\left(\theta_{\mathrm{SL} 0}\right)\right\}, \max \left\{\operatorname{SLL}\left(\theta_{\mathrm{SL} 5}\right)\right\},\right. \\
\left.\max \left\{\operatorname{SLL}\left(\theta_{\mathrm{SL} 10}\right)\right\}\right\}-S L_{T} \leq 0,
\end{gathered}
$$

where $\mathrm{SL}_{T}$ is the SLL target.

Now, we set up a model to be simulated by making use of MATLAB and the above mentioned equations (6), (7), (8), and (11) to compute the amplitude and phase of the voltage excitations for a given number of elements within a tilt range. As an example of simulation, 5 elements are used $\left(N=5 \rightarrow p_{i}=(2 i-N-1) / 2\right)$ and a number of 10 particles are initialized within their corresponding velocities. In (6), the inertia weight is set to 0.7 and the acceleration constants are set to 1.5. At each iteration step, the pattern is computed for three frequencies at different tilts. For each tilt, the maximum side lobe level is computed. Thus, three values (maximum level at $0^{\circ}, 5^{\circ}$, and $10^{\circ}$ ) are obtained to form a set based on which a maximum is selected as output. And the personal best position of each particle can be obtained along with the global best position. The process is summarized in the diagram shown in Figure 6.

After processing, the best output regarding the desired requirements (SLL not greater than $-15 \mathrm{~dB}$ ) is the particle with elements shown in Table 1.

And the representations of the vertical pattern of $0^{\circ}, 5^{\circ}$, and $10^{\circ}$ tilt according to the frequency are given in Figures $7(\mathrm{a}), 7(\mathrm{~b})$, and 7 (c) as benchmarked below. From the figures, we can observe good SLL suppression within the tilt range specified. And we can notice an increased lobe level especially around $10^{\circ}$ tilt surpassing the target. Such lobe particularly known also as grating lobe is related to the physical structure (distance between antenna elements) and can be object of further optimization issue.

\section{Experimental Results and Comments}

Motivated by the results from the above analysis, we aim to implement and test the antenna in this part. The amplitude and phase of the voltage excitation of the elements can be practically designed based on the parameters in Table 1 . To obtain the exact amplitude ratios as depicted in the first line of Table 1, a power splitting element should be carefully designed which is not always practically feasible. However, by using a set of quarter-wavelength transformers (using coaxial cables with appropriate impedance), such ratios can be approximated closely to those analytical ones shown in Table 1 (e.g., a ratio of $0.5: 0.8: 1: 0.8: 0.5$ can be practically realized) to excite each radiating element. Regarding the phases shown in the second line of Table 1, relevant lengths can be properly obtained from a careful calibrated cut. To enable electrical beam steering, phase shifters are needed. We make use of Tongyu's four-way phase shifter. For simplicity, the center-element is used as reference so its phase shift is set to zero when beam steering mechanism is applied for a given direction (such element is not connected to a phase shifter). In this case, if a positive phase shift $(+\Delta)$ is applied to the first element located at the right side of the reference, the same amount of phase is applied to its symmetric (element located at the left side) with a negative sign, that is, $-\Delta$. Accordingly, $+2 \Delta$ phase shift is applied to the second right side element while that one of its corresponding symmetric is $-2 \Delta$. Thus, for a selected steered angle, the corresponding four phases from the phase shifter are $-2 \Delta,-\Delta,+\Delta$, and $+2 \Delta$ (in other words, $\Delta$ represents the corresponding phase shift, and the coefficient in front of it is the shifted amount $f_{5}(i), i=$ $1, \ldots, 5$ as discussed in above analysis). The feeding network is implemented and baseline testing is conducted to tune 

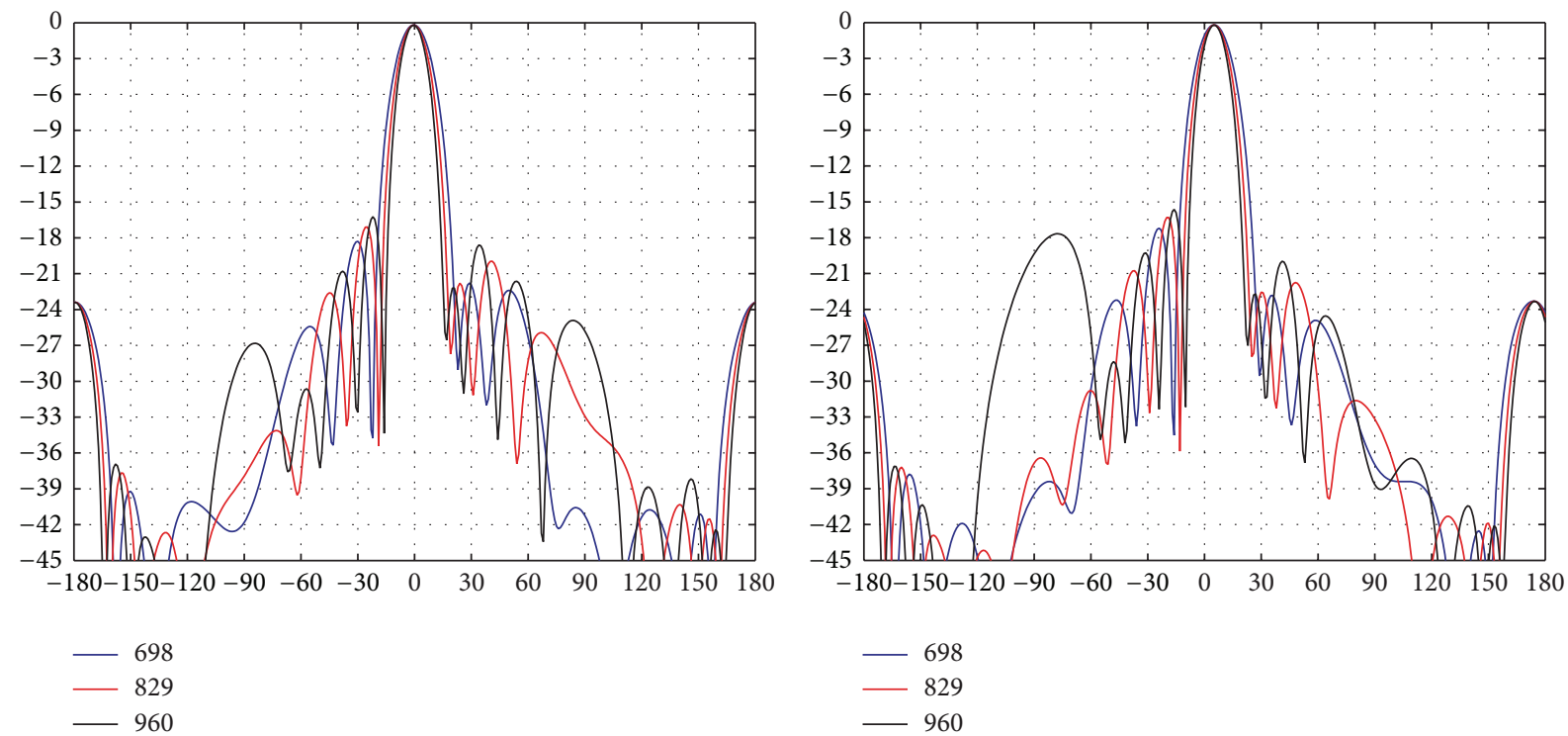

(a) Vertical pattern at $0^{\circ}$ tilt

(b) Vertical pattern at $5^{\circ}$ tilt

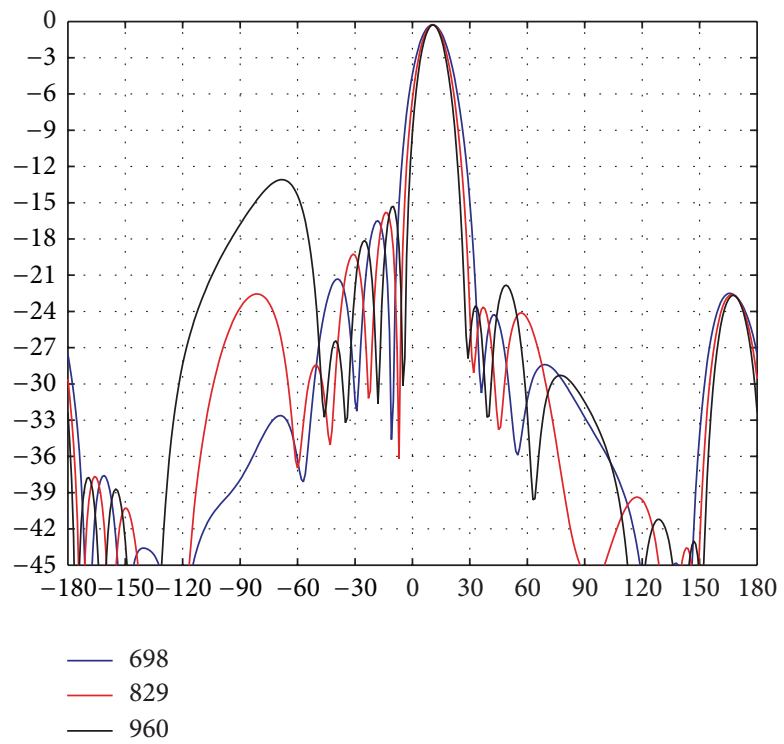

(c) Vertical pattern at $10^{\circ}$ tilt

FIGURE 7: Representation of the vertical pattern from PSO results according to the frequency at different tilts.

the VSWR $(<1.5)$ and port-to-port isolation $(<-25 \mathrm{~dB})$ using a network analyzer. The baseline parameters are depicted in Figure 8 at $0^{\circ}$ tilt (being the worst case observed during experiments). From the figure, we can realize a VSWR value of 1.3615 for one port and 1.3823 for the other port with an isolation of about $-27.052 \mathrm{~dB}$. After, far-field test within Tongyu's anechoic chamber is conducted as shown in Figure 9. The anechoic chamber having dimensions of $11 \times 11 \times 55 \mathrm{~m}$ represents the biggest one in China and has been used for several network operators for products approvals. The pattern test results are depicted in Figure 10. Figures 10(a), 10(b), and 10 (c) show the representation of the pattern at $0^{\circ}, 5^{\circ}$, and $10^{\circ}$ tilt, respectively, for one polarization (due to close similarity). The left part is the horizontal pattern while the right one represents the vertical pattern. Four frequencies are represented for clear displaying $(698 \mathrm{MHz}$ blue, $790 \mathrm{MHz}$ green, $880 \mathrm{MHz}$ red, and $960 \mathrm{MHz}$ black) but the test has shown a stable pattern across a wide frequency range as benchmarked in Table 2, for example. From the figures, we can observe good radiation characteristics from the horizontal plane where the copolar and cross-polar patterns are also given to realize good cross-polar discrimination ration at bore sight.

In addition, a stable $3 \mathrm{~dB}$ HPBW as shown in Table 2 for both polarizations where we can notice that more than $95 \%$ of the tested values satisfy the $90^{\circ} \pm 5^{\circ}$ as expected shows good antenna performances for mobile cellular applications. In the vertical plane, a good first side lobe control can be seen from the figures with a level less than $15 \mathrm{~dB}$ as targeted. 


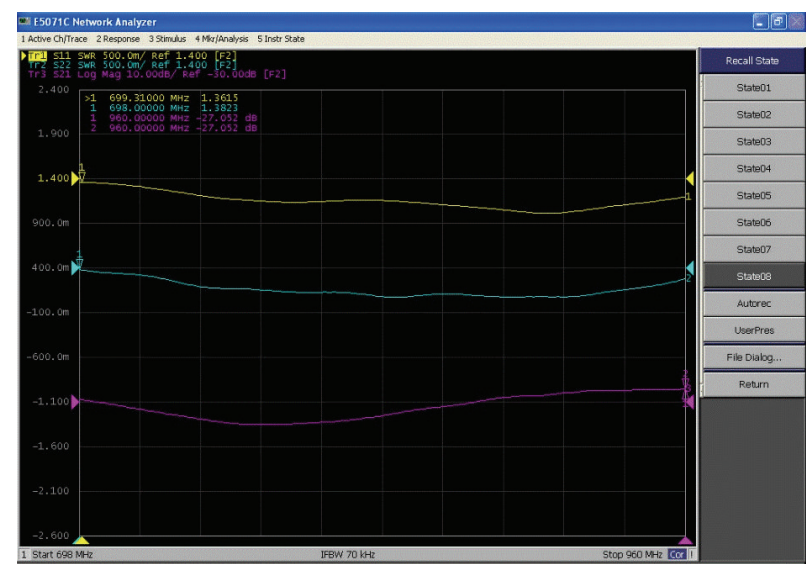

FIGURE 8: Representation of VSWR and isolation according to frequency.

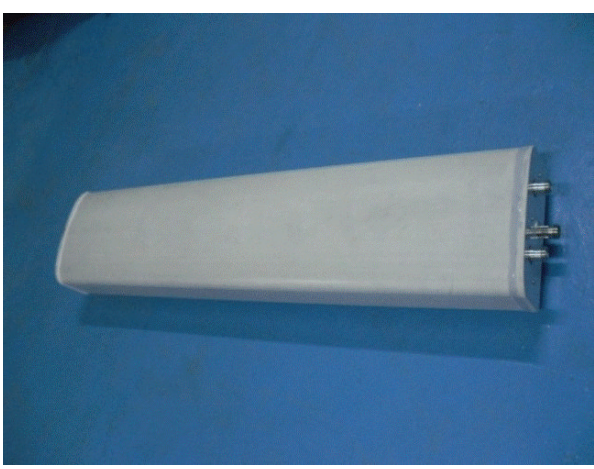

(a)

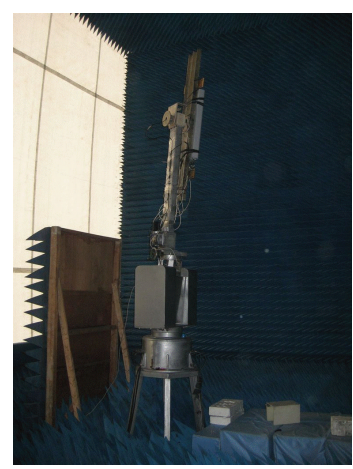

(b)

FIgURE 9: Assembled antenna and its far-field pattern testing environment.

TABLE 2: HPBW of the two ports $(+45$ and -45$)$ according to the frequency at different tilts.

\begin{tabular}{|c|c|c|c|c|c|c|}
\hline \multirow[t]{2}{*}{ Frequency $(\mathrm{MHz})$} & \multicolumn{2}{|c|}{$\begin{array}{c}0^{\circ} \text { tilt } \\
\operatorname{HPBW}\left({ }^{\circ}\right)\end{array}$} & \multicolumn{2}{|c|}{$\begin{array}{c}5^{\circ} \text { tilt } \\
\text { HPBW }\left(^{\circ}\right)\end{array}$} & \multicolumn{2}{|c|}{$\begin{array}{c}10^{\circ} \text { tilt } \\
\operatorname{HPBW}\left({ }^{\circ}\right)\end{array}$} \\
\hline & $+45^{\circ}$ & $-45^{\circ}$ & $+45^{\circ}$ & $-45^{\circ}$ & $+45^{\circ}$ & $-45^{\circ}$ \\
\hline 698 & 86.66 & 85.87 & 88.64 & 86.79 & 88.05 & 86.72 \\
\hline 720 & 85.06 & 86.38 & 85.60 & 86.39 & 86.96 & 85.02 \\
\hline 750 & 86.66 & 87.13 & 86.27 & 88.32 & 86.23 & 89.74 \\
\hline 780 & 87.73 & 89.35 & 89.08 & 89.43 & 88.48 & 89.35 \\
\hline 790 & 89.03 & 90.11 & 89.33 & 90.25 & 89.62 & 90.00 \\
\hline 806 & 88.01 & 87.30 & 88.37 & 89.58 & 90.96 & 89.81 \\
\hline 824 & 88.22 & 90.30 & 88.54 & 91.72 & 90.10 & 91.62 \\
\hline 840 & 89.94 & 91.91 & 89.07 & 92.88 & 89.02 & 93.28 \\
\hline 860 & 88.71 & 91.61 & 90.05 & 94.31 & 91.01 & 93.73 \\
\hline 880 & 89.35 & 90.92 & 90.93 & 92.17 & 93.44 & 91.66 \\
\hline 890 & 89.33 & 92.74 & 90.37 & 93.39 & 92.16 & 92.47 \\
\hline 896 & 89.54 & 91.48 & 89.38 & 92.17 & 90.58 & 93.08 \\
\hline 915 & 90.45 & 89.52 & 91.52 & 90.35 & 92.14 & 91.45 \\
\hline 930 & 93.49 & 95.08 & 94.15 & 93.75 & 91.11 & 94.70 \\
\hline 940 & 90.67 & 93.54 & 92.12 & 92.80 & 87.98 & 97.30 \\
\hline 960 & 91.69 & 92.85 & 96.97 & 96.12 & 94.43 & 97.66 \\
\hline
\end{tabular}



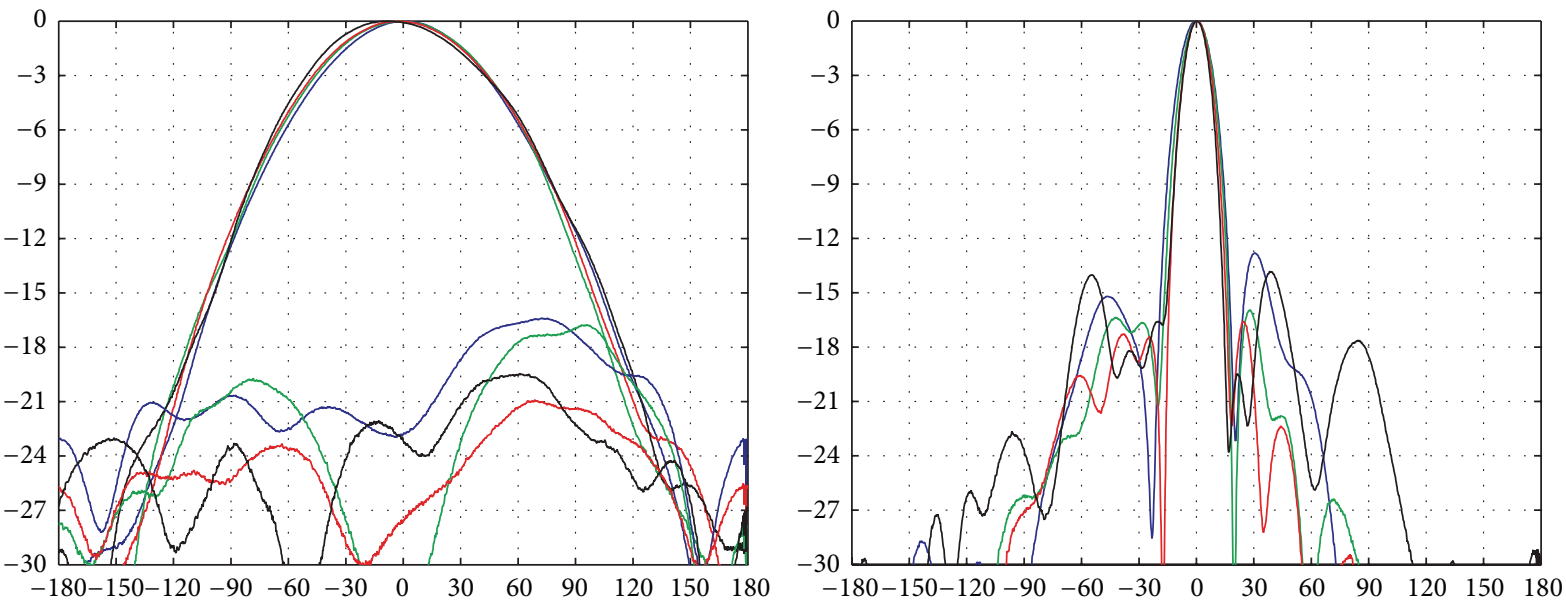

(a) Horizontal (left) and vertical (right) patterns at $0^{\circ}$ tilt
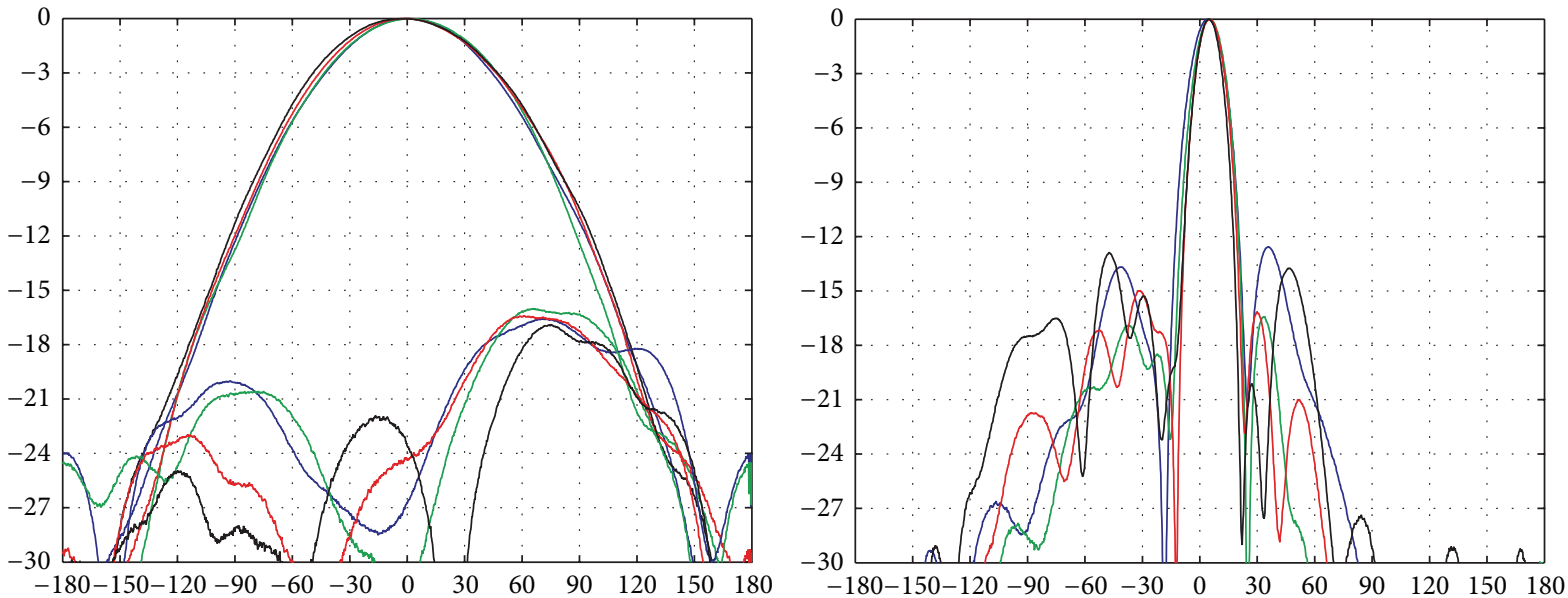

(b) Horizontal (left) and vertical (right) patterns at $5^{\circ}$ tilt
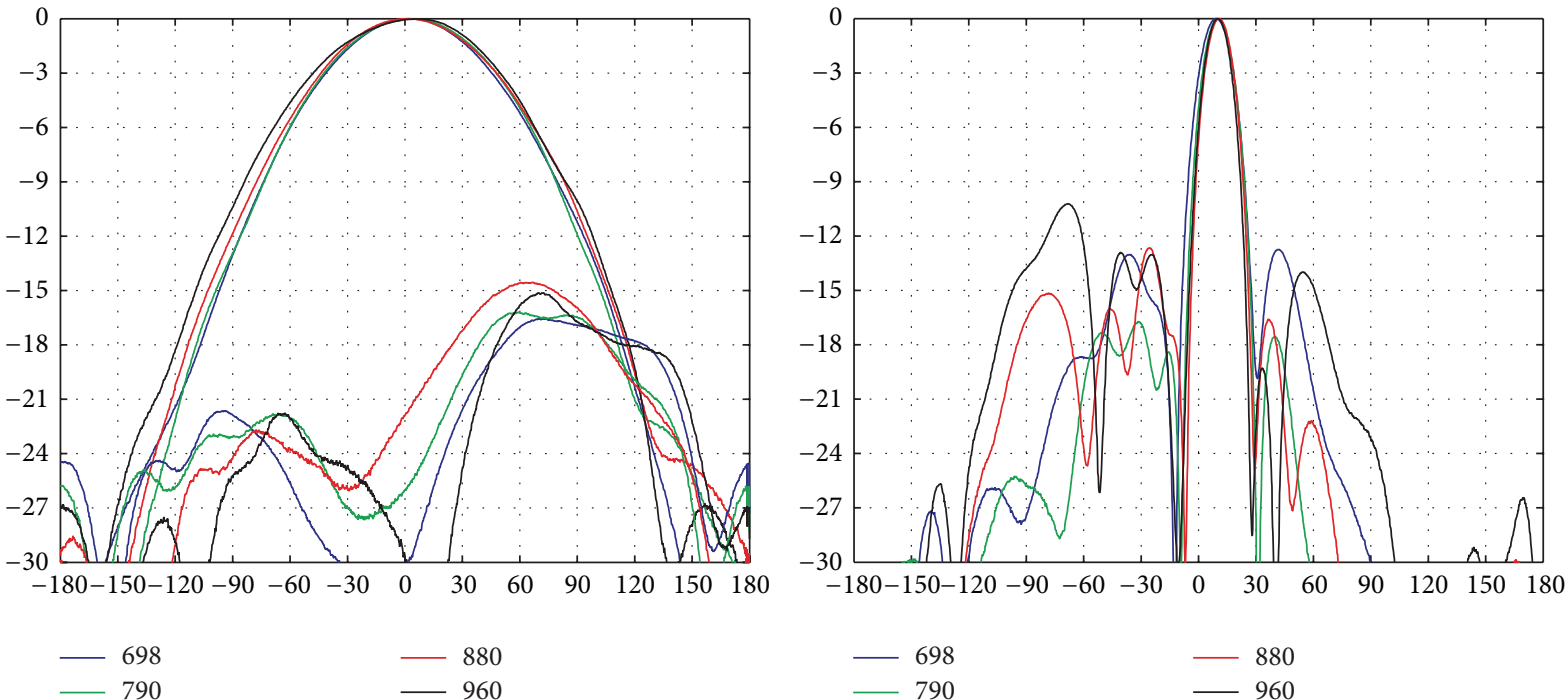

(c) Horizontal (left) and vertical (right) patterns at $10^{\circ}$ tilt

FIGURE 10: Representation of the measured horizontal (left) and vertical (right) patterns according to the frequency (698 MHz blue, $790 \mathrm{MHz}$ green, $880 \mathrm{MHz}$ red, and $960 \mathrm{MHz}$ black) at different tilts. 


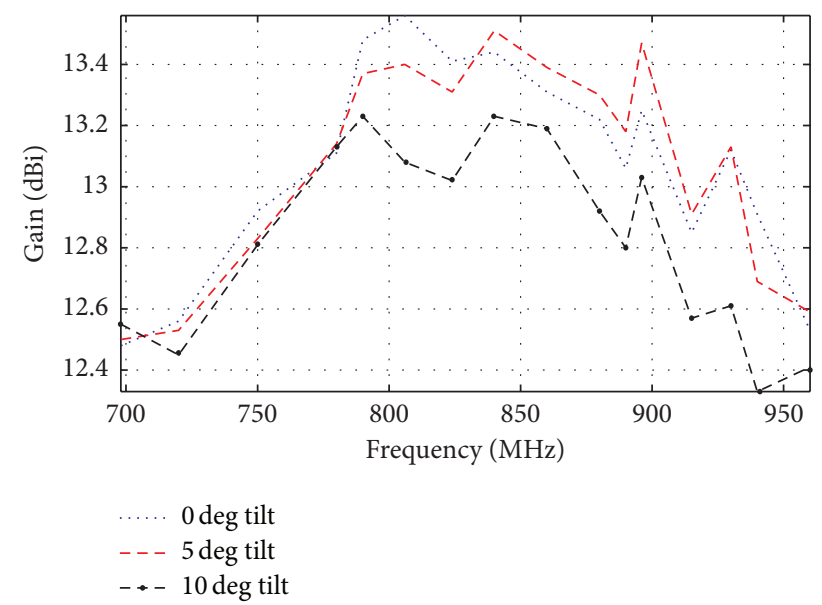

FIGURE 11: Representation of gain according to frequency at different tilt.

However, secondary lobes appear when tilting and more precisely at $10^{\circ}$ where a grating lobe of about $10 \mathrm{~dB}$ level is obtained. In fact, the antenna tilt angle can be defined as the angle between the center direction of the main beam and the azimuthal (also called horizontal) direction. Besides such main beam, there are also additional secondary beams (called side lobes). And the side lobe levels depend strongly on the excitation (amplitude and phase) mechanism used to feed the different elements progressively. And the phase shifts become sensible according to the tilt angle. Roughly speaking, the more you tilt the more the side lobe levels grow resulting in decreasing the performances. Thus, practically, the electrical tilt is generally limited due to changing side lobe level. It is important to note that the errors from the feeding networks due to cable lengths affect the phase of the signal of interest impacting on the vertical pattern as well. The measured antenna maximum gain is more than $13.5 \mathrm{dBi}$ as can be observed from Figure 11. For higher gain applications, adding extra radiating elements is necessary. However, in certain cases, the antenna has limited size constraint resulting in being not easy to use the exact number of elements required to output the desired gain from the array theory. To this end, one can think about reducing the spacing between elements which may affect the cross coupling, thus impacting the uplink diversity, for example. An alternative way to process is to reduce the $3 \mathrm{~dB}$ HPBW.

\section{Conclusion}

The fastest emerging wireless access generates numerous challenges on the antenna systems design as new frequency bands should be fully covered to meet the demands for various application levels in terms of radio network design offering, for example, high in-building penetration. This work designed and developed a new dual-polarized wideband base station antenna supporting $698 \mathrm{MHz}$ to $960 \mathrm{MHz}$ with a azimuth-plan half-power beam width of $90^{\circ}$ covering all mainstream LTE $700 / 800 / 900 \mathrm{MHz}$ frequency bands representing the widest low frequency range being actively used in the current mobile communication industry. Rigorous algorithm based on swarm method was developed to control the structure and tune the electrical performances under strict base station antenna requirements. Experimental results from pattern tests demonstrate the design analysis and the significant advantages of using swarm method in the antenna development process.

\section{Conflict of Interests}

The authors declare that there is no conflict of interests regarding the publication of this paper.

\section{References}

[1] A. Goldsmith, Wireless Communications, Cambridge University Press, Cambridge, UK, 2005.

[2] J. G. Andrews, "Interference cancellation for cellular systems: a contemporary overview," IEEE Wireless Communications, vol. 12, no. 2, pp. 19-29, 2005.

[3] P. Li, C. Zhang, and Y. Fang, "The capacity of wireless ad hoc networks using directional antennas," IEEE Transactions on Mobile Computing, vol. 10, no. 10, pp. 1374-1387, 2011.

[4] D. Samb and L. Yu, "Performance analysis of amplify and forward cooperative relaying protocol in wireless communication system," Wireless Personal Communications, vol. 70, no. 2, pp. 969-983, 2013.

[5] L. Qu, J. He, and C. Assi, "Understanding the benefits of successive interference cancellation in multi-rate multi-hop wireless networks," IEEE Transactions on Communications, vol. 62, no. 7, pp. 2465-2477, 2014.

[6] M. Deruyck, W. Joseph, B. Lannoo, D. Colle, and L. Martens, "Designing energy-efficient wireless access networks: LTE and LTE-advanced," IEEE Internet Computing, vol. 17, no. 5, pp. 3945, 2013.

[7] C. E. Shannon, "A mathematical theory of communication," The Bell System Technical Journal, vol. 27, pp. 379-423, 1948.

[8] S. Forconi and A. Vizzarri, "Review of studies on end-to-end QoS in LTE networks," in Proceedings of the AEIT Annual Conference, pp. 1-6, IEEE, Mondello, Italy, October 2013.

[9] S. Koziel, S. Ogurtsov, W. Zieniutycz, and A. Bekasiewicz, "Design of a planar UWB dipole antenna with an integrated balun using surrogate-based optimization," IEEE Antennas and Wireless Propagation Letters, vol. PP, no. 99, 2014.

[10] N. K. Kiem, H. N. B. Phuong, and D. N. Chien, "Design of compact $4 \times 4$ UWB-MIMO antenna with WLAN band rejection," International Journal of Antennas and Propagation, vol. 2014, Article ID 539094, 11 pages, 2014.

[11] J. Robinson and Y. Rahmat-Samii, "Particle swarm optimization in electromagnetics," IEEE Transactions on Antennas and Propagation, vol. 52, no. 2, pp. 397-407, 2004.

[12] N. Jin and Y. Rahmat-Samii, "Advances in particle swarm optimization for antenna designs: real-number, binary, singleobjective and multiobjective implementations," IEEE Transactions on Antennas and Propagation, vol. 55, no. 3, pp. 556-567, 2007.

[13] J. D. Kraus, R. J. Marhefka, and A. S. Khan, Antennas for All Applications, New York, NY, USA, McGraw Hill, 3rd edition, 2002. 
[14] D. Samb, Z. Wu, and M. Liu, "Development of wideband base station antenna based on particle swar method for LTE networks," Antenna Systems and Technology Magazine. In press.

[15] P. S. Kildal, Foundations of Antennas A Unified Approach, Studentlitteratur, 2002.

[16] R. A. Rutenbar, "Simulated annealing algorithms: an overview," IEEE Circuits and Devices Magazine, vol. 5, no. 1, pp. 19-26, 1989.

[17] W. Zijun and M. Kolonko, "Asymptotic properties of a generalized cross-entropy optimization algorithm," IEEE Transactions on Evolutionary Computation, vol. 18, no. 5, pp. 658-673, 2014.

[18] J. Kennedy and R. Eberhart, "Particle swarm optimization," in Proceedings of the IEEE International Conference on Neural Networks, pp. 1942-1948, December 1995.

[19] K. Deb and H. Jain, "An evolutionary many-objective optimization algorithm using reference-point- based nondominated sorting approach, part I: solving problems with box constraints," IEEE Transactions on Evolutionary Computation, vol. 18, no. 4, pp. 577-601, 2014.

[20] S. Song and R. D. Murch, "An efficient approach for optimizing frequency reconfigurable pixel antennas using genetic algorithms," IEEE Transactions on Antennas and Propagation, vol. 62, no. 2, pp. 609-620, 2014. 

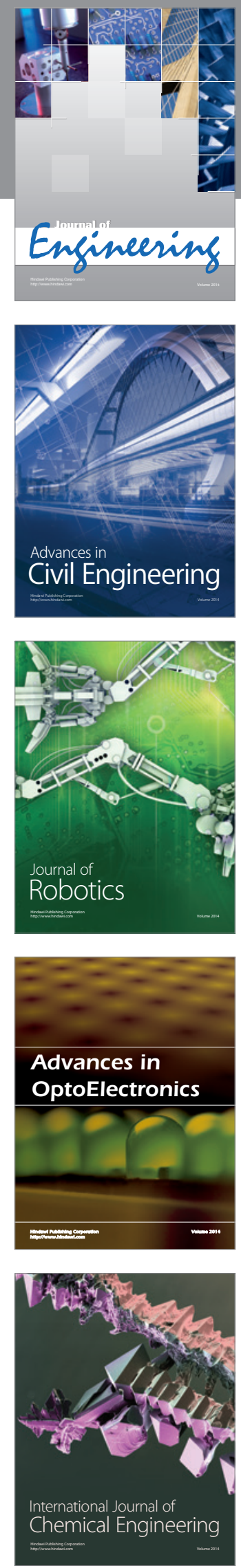

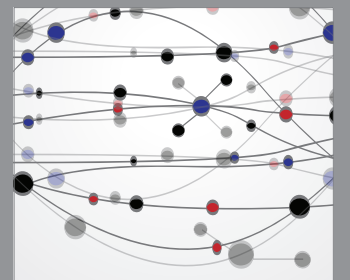

The Scientific World Journal
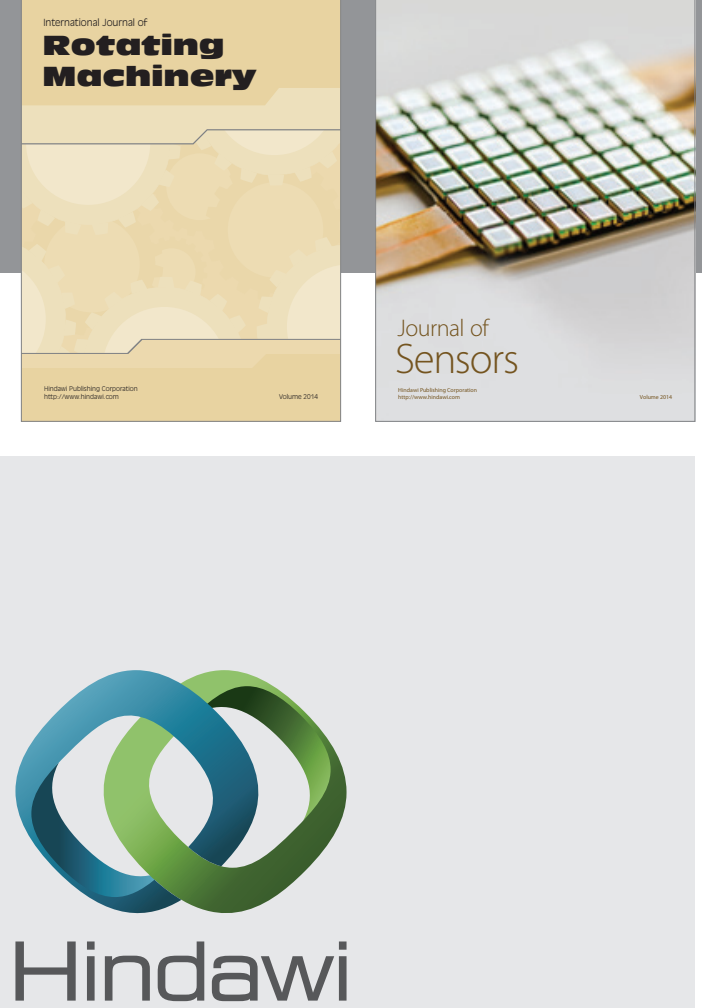

Submit your manuscripts at http://www.hindawi.com
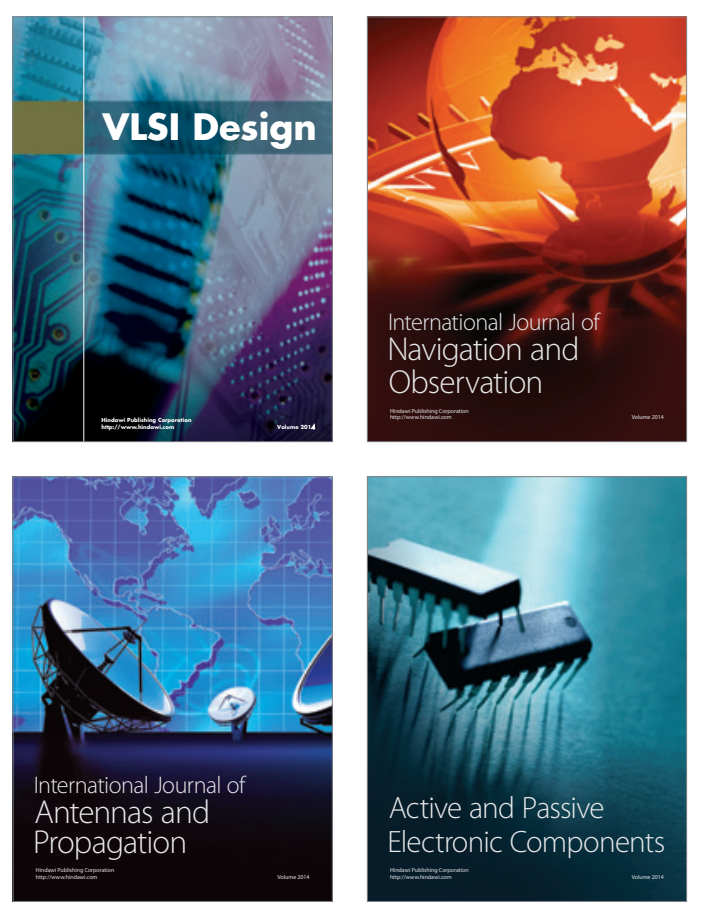
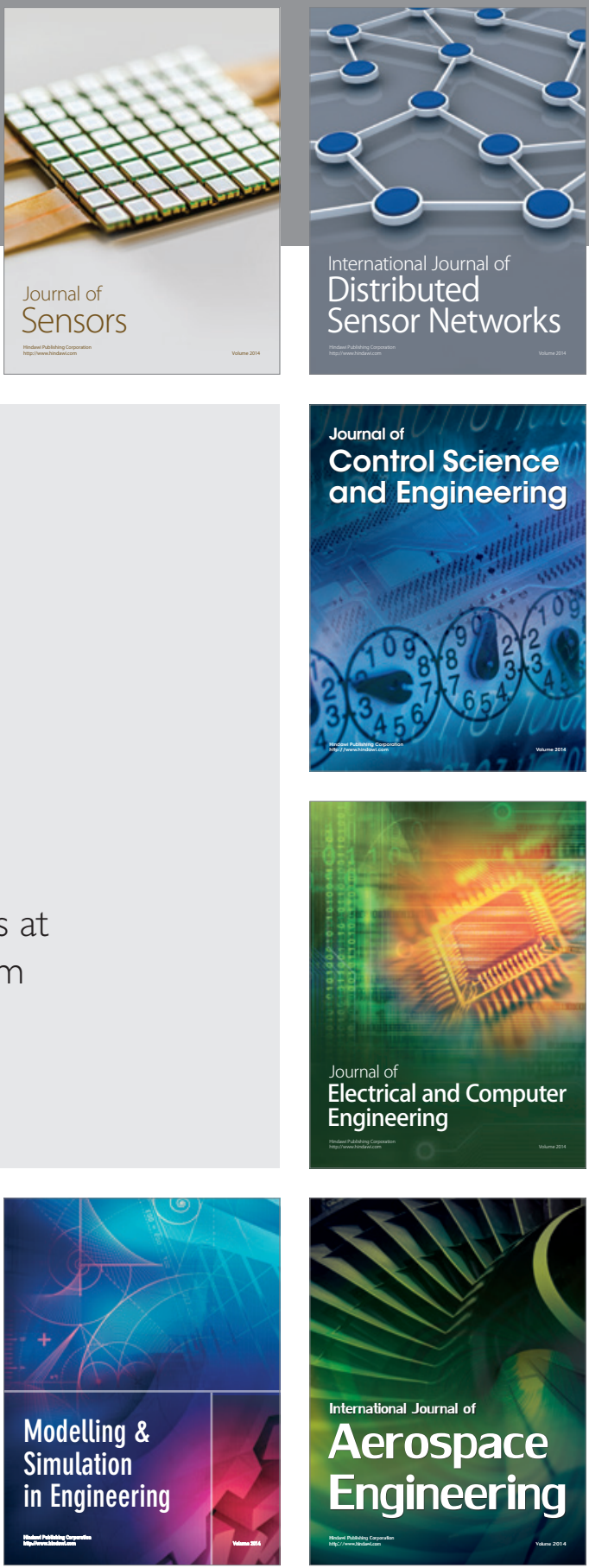

Journal of

Control Science

and Engineering
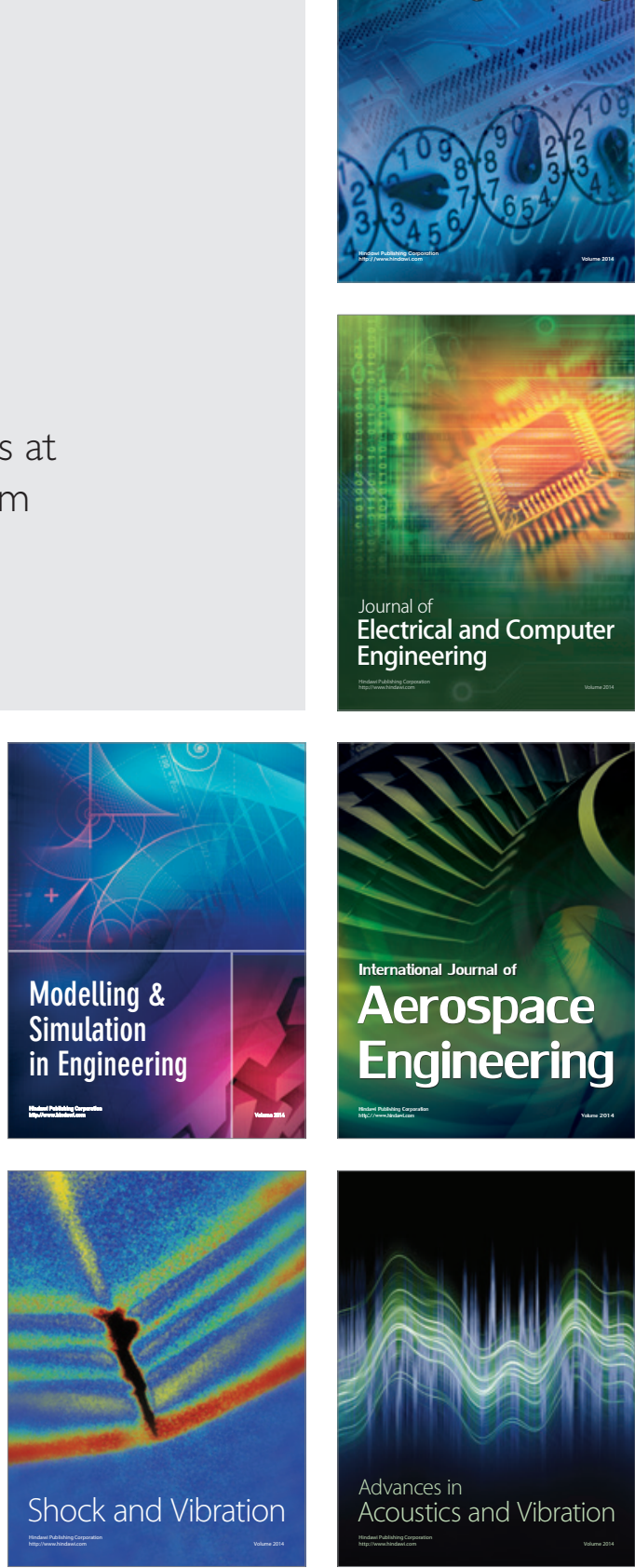